Service social

\title{
Représentations sociales du genre chez de jeunes adultes québécois : analyse exploratoire de la validité du Bem Sex Role Inventory
}

\author{
Mathieu Philibert, Andréanne Gagné, Émilie Blanchette et Line Chamberland
}

Volume 63, numéro 2, 2017

Genres et sexualités chez les jeunes

URI : https://id.erudit.org/iderudit/1046504ar

DOI : https://doi.org/10.7202/1046504ar

Aller au sommaire du numéro

Éditeur(s)

École de travail social et de criminologie de l’Université Laval

ISSN

1708-1734 (numérique)

Découvrir la revue

Citer cet article

Philibert, M., Gagné, A., Blanchette, É. \& Chamberland, L. (2017).

Représentations sociales du genre chez de jeunes adultes québécois : analyse exploratoire de la validité du Bem Sex Role Inventory. Service social, 63(2),

132-142. https://doi.org/10.7202/1046504ar
Résumé de l'article

Le genre, qui fait notamment référence aux rôles, comportements et identités des individus, fait l'objet de représentations sociales souvent stéréotypées. L'adhésion aux stéréotypes de genre est associée à la santé et au bien-être des personnes, notamment celles issues de groupes minorisés. Cette étude visait à évaluer la validité d'une mesure de l'adhésion aux stéréotypes de genre, le Bem Sex Role Inventory. Selon les données recueillies auprès d'une population étudiante, cette mesure ne correspondrait pas aux représentations des rôles de genre des jeunes adultes interrogés, et ce, bien que le genre demeure un élément structurant des représentations normatives des rôles, comportements, expressions et identités. 


\title{
REPRÉSENTATIONS SOCIALES DU GENRE CHEZ DE JEUNES ADULTES QUÉbÉCOIS : ANALYSE EXPLORATOIRE DE LA VALIDITÉ DU BEM SEX ROLE INVENTORY
}

\author{
PHILIBERT, Mathieu, Ph. D. santé publique \\ GAGNÉ, Andréanne, B.A. (c) sexologie \\ BLANCHETTE, Émilie, B.A. (c) sexologie \\ CHAMBERLAND, Line, Ph. D. sociologie
}

\section{RÉSUMÉ}

Le genre, qui fait notamment référence aux rôles, comportements et identités des individus, fait l'objet de représentations sociales souvent stéréotypées. L'adhésion aux stéréotypes de genre est associée à la santé et au bien-être des personnes, notamment celles issues de groupes minorisés. Cette étude visait à évaluer la validité d'une mesure de l'adhésion aux stéréotypes de genre, le Bem Sex Role Inventory. Selon les données recueillies auprès d'une population étudiante, cette mesure ne correspondrait pas aux représentations des rôles de genre des jeunes adultes interrogés, et ce, bien que le genre demeure un élément structurant des représentations normatives des rôles, comportements, expressions et identités.

\begin{abstract}
Gender, which refer namely to individual roles, behaviours and identities, is often subject to stereotyped social representation. Adherence to gender stereotypes is associated to individuals' health and wellbeing, namely among individuals from minority groups. This study aimed at evaluating the validity of a measure of adherence to gender stereotypes, the Bem Sex Role Inventory. The data collected from a student population suggest that this measure does not correspond to the gender roles' representations of young adults who participated in our study and that, despite the fact that gender remains an aspect that has a structuring effect on the normative representations of roles, behaviours, expressions and identities.
\end{abstract}




\section{INTRODUCTION}

Le genre fait référence aux «rôles, comportements, expressions et identités » définis socialement (Conseil de recherches en sciences humaines du Canada, Conseil de recherches en sciences naturelles et en génie du Canada et Instituts de recherche en santé du Canada, 2014). Ces rôles, comportements, expressions et identités attribués au genre varient selon le contexte social, géographique et historique. Alors que le genre a longtemps été associé au sexe, cette association est aujourd'hui remise en question, notamment en ce qui a trait aux représentations conventionnelles qui prescrivent, dans un modèle binaire cisnormatif, des rôles, des comportements et des attitudes en fonction du sexe assigné à la naissance. Malgré la démonstration d'une grande diversité des expressions et des identités individuelles (Spence, 1993 ; Spence et Hall, 1996), les représentations sociales de genre demeurent stéréotypées.

Les stéréotypes de genre désignent les représentations attribuées à des individus selon leur appartenance à un groupe, dans ce cas-ci leur appartenance à l'une ou l'autre des catégories de genre (Plante, Théorêt et Favreau, 2010). Ils constituent des prescriptions sociales en matière de rôles, de comportements et d'attitudes associées au sexe des personnes. Les stéréotypes de genre qui ont cours dans un contexte donné sont susceptibles d'affecter les attitudes et les comportements des individus qui y adhèrent. Par exemple, plusieurs études ont mesuré l'adhésion aux stéréotypes associant de meilleures compétences en mathématiques aux garçons et de meilleures compétences en français aux filles et ont tenté d'en évaluer les impacts sur le rendement scolaire par le biais de variables médiatrices telles que la perception qu'a la personne de sa compétence en ces matières, l'anxiété générée par la situation et la valeur accordée aux apprentissages dans l'une ou l'autre matière (Plante et al., 2010). II faut cependant prendre garde, selon ces auteures, à ne pas présumer des répercussions des stéréotypes de genre, mais plutôt prendre la mesure de l'adhésion à de tels stéréotypes (ce qui soulève la question des divers outils de mesure), dans des contextes spécifiques, et analyser la relation entre les stéréotypes de genre et leurs conséquences sur les individus telle que médiatisée par l'adhésion subjective à ces stéréotypes.

Galambos, Berenbaum et McHale (2009) proposent d'examiner le développement du genre à l'adolescence à travers une matrice de concepts parmi lesquels on retrouve les conceptions relatives au genre, comme la connaissance des stéréotypes de genre et les croyances quant au caractère approprié ou non d'activités, de capacités, de comportements et de rôles en fonction du genre. Ces conceptions constituent une dimension importante du développement du genre. Cette dimension peut être mise en correspondance avec l'identité ou la perception de soi, avec les préférences subjectives et avec les comportements et agissements dans diverses sphères de vie.

Par ailleurs, diverses études ont exploré les liens entre l'adhésion aux stéréotypes de genre et certains indicateurs de santé dans la population des jeunes adultes ou chez certains groupes minorisés comme les personnes issues de la diversité sexuelle. Ainsi, l'adhésion aux stéréotypes de genre serait un facteur de protection contre les symptômes de dépression chez les jeunes femmes adultes (Gibson, Baker et Milner, 2016). Chez les jeunes hommes, toutefois, cet effet protecteur contre les symptômes de dépression de l'adhésion aux stéréotypes de genre dépendrait du niveau d'éducation : il serait présent chez les jeunes hommes ayant un niveau d'éducation collégial ou supérieur, mais pas chez ceux ayant un niveau d'éducation plus faible (Gibson et al., 2016). On a aussi observé une variation selon le genre de l'effet de l'adhésion aux stéréotypes de genre sur des comportements autodestructeurs. Chez les jeunes femmes, l'adhésion aux stéréotypes de la féminité réduirait l'adoption 
de comportements autodestructeurs, alors que chez les jeunes hommes, l'adhésion aux stéréotypes de la masculinité contribuerait à augmenter le risque d'adopter des comportements autodestructeurs (Tsirigotis, Gruszczynski et Tsirigotis-Maniecka, 2013). Des comportements non conformes aux rôles de genre sont associés à un accroissement du risque de victimisation par les pairs et de suicide chez les jeunes homosexuels (Friedman et al., 2006 ; Fitzpatrick et al., 2005). Cela dit, l'influence de la nonadhésion aux stéréotypes de genre sur les symptômes suicidaires des jeunes issus de minorités sexuelles est grandement tributaire du soutien par la famille et par les pairs (Fitzpatrick et al., 2005).

Plusieurs instruments de mesure du degré d'adhésion aux stéréotypes de genre ont été proposés. Le Bem Sex Role Inventory (Bem, 1974) figure parmi les plus couramment utilisés (Plante et al., 2010). Cet instrument reconnait l'existence de genres (c.-à-d. masculin ou féminin), mais s'éloigne d'une perspective binaire en permettant qu'un homme ou une femme puisse à la fois posséder des traits masculins, féminins ou neutres (androgynes). À partir d'un ensemble de 60 traits de personnalité préalablement associés à un genre (20 traits masculins, 20 traits féminins et 20 traits neutres), les personnes décrivent dans quelle mesure elles estiment correspondre à chacun des traits. Sur la base de ces réponses, trois scores sont produits par l'instrument, soit un pour la masculinité, un pour la féminité et un autre pour «l'androgynie » (neutralité). Plus une personne estime qu'elle correspond à des traits considérés féminins, plus son score de féminité sera élevé, par exemple. Il en va de même pour les traits masculins et pour les traits neutres.

Ainsi, la mesure d'adhésion aux stéréotypes de genre est fondée sur une classification préalable des traits qui composent l'instrument de mesure (tableau 1). Cette classification des traits (masculins, féminins ou neutres) est le résultat d'une enquête menée auprès d'étudiant.e.s de l'Université Stanford aux États-Unis en 1972. Les répondant.e.s devaient indiquer dans quelle mesure, selon eux, un trait de personnalité était valorisé pour un homme ou pour une femme dans la société américaine (la procédure est décrite en détail dans la section « Méthodes » ci-dessous). Avec cet instrument de mesure, Bem cherchait à identifier des traits de personnalité qui décrivent la féminité, la masculinité et l'androgynie, et ce, tant chez les femmes que chez les hommes. Toutefois, comme le Bem Sex Role Inventory (BSRI) fait appel aux perceptions sociales, et parce que le genre se réfère à un concept social, changeant, complexe et multidimensionnel, le BSRI est susceptible d'être sensible au contexte de son utilisation. À notre connaissance, aucune étude sur la validité du BSRI n'a été faite auprès des jeunes adultes québécois. Dans cet article, nous décrivons les résultats d'une étude exploratoire visant à évaluer si la classification des items du BSRI correspond aux représentations sociales de genre de jeunes adultes québécois en 2017 et si ces représentations varient selon le sexe ou l'orientation sexuelle.

\section{MÉTHODES}

Notre enquête sur les représentations sociales de genre a eu lieu à l'Université du Québec à Montréal (UQAM) et s'adressait à tous.tes les étudiant.e.s fréquentant l'établissement, indépendamment de leur programme, régime ou cycle d'études. Le recrutement s'est fait avec le concours de toutes les associations étudiantes facultaires de l'université, à l'exception d'une seule. Ces associations ont envoyé au moins deux courriels d'invitation à l'ensemble de leurs membres. Le questionnaire a été disponible en ligne du 20 février 2017 au $1^{\text {er }}$ mai 2017.

L'enquête et l'analyse des résultats visaient à répliquer la démarche de Bem (1974). Le Bem Sex Role Inventory (BSRI) a été construit en trois étapes. Premièrement, l'équipe de recherche a identifié 200 traits de personnalité considérés comme masculins ou féminins ainsi que 200 traits considérés 
comme neutres. Deuxièmement, ces 400 traits ont été soumis à 100 juges ( 50 hommes et 50 femmes) recrutés sur le campus de l'Université Stanford (États-Unis) en 1972. Les juges devaient indiquer dans quelle mesure un trait de personnalité était, selon eux, valorisé comme un trait masculin ou comme un trait féminin dans la société américaine, sur une échelle de 1 («Pas du tout») à 7 (« Tout à fait »). Un sous-groupe formé de la moitié des juges devait se prononcer au sujet des femmes seulement («In American society, how desirable is it for a woman to be... ») et l'autre sous-groupe de juges, au sujet des hommes seulement ("In American society, how desirable is it for a man to be... »). Troisièmement, pour chacun des 400 traits évalués, un test statistique (test $t$ ) a été utilisé pour déterminer si les scores moyens des deux sous-groupes de répondant.e.s étaient différents. Lorsqu'une différence significative était observée, un trait était considéré comme masculin si le score moyen le plus élevé avait été obtenu par le sous-groupe de juges répondant au sujet des hommes tandis qu'un trait était considéré féminin si le score moyen le plus élevé avait été obtenu par le sous-groupe de juges répondant au sujet des femmes. Lorsque les scores moyens n'étaient pas différents statistiquement, un trait était considéré comme neutre (lorsque $t<1,2$ ). Parmi les 400 traits analysés, 60 ont été retenus, soit 20 traits masculins, 20 traits féminins et 20 traits neutres. Les 400 traits originellement évalués ne sont pas décrits dans l'article de Bem (1974); seuls les 60 traits retenus sont présentés (tableau 1). Notre étude n'a donc pas pu reproduire intégralement la démarche de Bem (1974). Ce sont les 60 traits retenus dans l'instrument qui ont été repris dans l'étude menée à l'UQAM. Leur traduction est présentée au tableau 1.

Les participant.e.s à notre étude étaient distribués aléatoirement entre l'un et l'autre des sous-groupes de juges : la moitié des participant.e.s devaient évaluer dans quelle mesure les traits sont valorisés pour un homme et l'autre moitié devait évaluer dans quelle mesure les traits sont valorisés pour une femme. À l'instar de l'étude de Bem (1974), les questions ont été formulées ainsi : « Dans la société québécoise, à quel point les caractéristiques suivantes sont-elles valorisées pour les femmes? 》 ou « Dans la société québécoise, à quel point les caractéristiques suivantes sont-elles valorisées pour les hommes? ». La distribution aléatoire entre les deux sous-groupes de juges visait à augmenter la probabilité d'obtenir une proportion égale de réponses au sujet des hommes et des femmes. Également comme dans l'étude de Bem, les participant.e.s devaient indiquer leur perception pour chacun des 60 traits sur une échelle de type Likert ( 1 = Pas du tout en accord ; $7=$ Tout à fait en accord). Conformément à la démarche utilisée par Bem, les traits étaient classés comme féminins ou masculins si une différence significative était observée entre les scores des sous-groupes de répondant.e.s sur la base d'un test $t$. Les autres traits, ceux pour lesquels aucune différence significative n'était observée, ont été classés comme neutres. La variation des représentations sociales de genre selon le genre ou l'orientation sexuelle a été évaluée à l'aide du coefficient de kappa (к), une mesure de concordance de classifications. Une pondération a été utilisée pour corriger la surreprésentation des femmes dans l'échantillon : un poids plus faible (77/255) a été associé à chacune des femmes de l'échantillon $(n=255)$ afin que la somme des poids de l'échantillon féminin corresponde à la somme des poids de l'échantillon masculin $(n=77$, poids $=1$ ).

\section{RÉSUltats}

Au total, 332 jeunes adultes cisgenres (c'est-à-dire dont l'identité de genre correspond au sexe/genre qui leur a été assigné à la naissance) âgés de 18 à 25 ans ont rempli le questionnaire. Ce groupe est composé de répondant.e.s qui se sont identifiés comme «femme » $(n=255)$ ou comme « homme » 
$(n=77)$. Cinq personnes s'identifiant comme non binaires (c'est-à-dire qui ne se sentent pas d'un genre en particulier ou qui contestent le fait que le genre se résume à deux catégories mutuellement exclusives) ou non cisgenres ont été exclues des analyses pour des raisons statistiques : leur nombre était trop petit pour constituer un groupe qui pouvait être comparé à un autre groupe. À la question "Vous vous considérez comme étant :... ? (réponses possibles : Hétérosexuel.le / Homosexuel.le, gai.e, lesbienne / Bisexuel.le / Bi-spirituel.le / Queer, pansexuel.le, allosexuel.le / Asexuel.le / Incertain.e / En questionnemement / Je préfère ne pas répondre / Autre), près du quart des répondant.e.s se sont dits non hétérosexuels ( 61 femmes et 18 hommes).

Le tableau 1 présente la classification des items résultant des réponses fournies par l'ensemble des répondant.e.s, de même que les classifications obtenues par sous-groupes de genre et d'orientation sexuelle des répondant.e.s. Dans l'ensemble, la classification issue des réponses fournies par les répondant.e.s de l'UQAM concorde plutôt bien avec celle du BSRI ( $\kappa=0,70$; Landis et Koch, 1977) : les traits considérés comme féminins ou masculins sont à peu près les mêmes dans les deux études. Par contre, le groupe des répondant.e.s de l'UQAM dans son ensemble classe différemment 12 des 20 traits considérés comme neutres par le BSRI, 11 de ceux-ci étant considérés comme féminins et un comme masculin (tableau 1).

L'analyse des résultats par genre des répondant.e.s (tableau 1) révèle que les femmes répondent de façon très similaire à l'ensemble des répondant.e.s de l'UQAM $(\kappa=0,98)$ : les traits considérés comme masculins ou féminins par le BSRI sont considérés par elles de la même façon, mais 12 traits neutres selon le BSRI sont plutôt considérés comme des traits féminins et deux traits neutres selon le BSRI sont plutôt considérés comme masculins. Ce résultat pourrait être un artifice de la surreprésentation des femmes dans l'échantillon. En effet, la distribution aléatoire des répondant.e.s entre chacun des sousgroupes de juges (l'un se prononçant au sujet des femmes et l'autre au sujet des hommes) a fait que les femmes se retrouvaient dans des proportions similaires $\left(\chi^{2}=0,286, p=0,593\right)$ dans chacun des sous-groupes de juges $(78,0 \%$ et $75,5 \%)$, ce qui limite l'effet de la pondération utilisée.

Les perceptions des hommes sont moins genrées que celles des femmes. Ils considèrent que trois traits féminins et trois traits masculins selon le BSRI seraient plutôt des traits neutres. Parmi les traits neutres du BSRI, les hommes considèrent que sept traits seraient plutôt féminins et un trait plutôt masculin. La classification des items neutres des femmes n'est que modérément concordante avec celle des hommes ( $\kappa=0,50$; Landis et Koch, 1977). Comparativement aux hommes, les femmes perçoivent davantage de traits qui sont neutres selon le BSRI comme étant genrés, considérant 12 de ceux-ci comme féminins et un comme masculin. Précisons encore que l'ensemble des traits neutres selon le BSRI qui sont considérés comme genrés par les hommes le sont aussi par les femmes et que pour ces traits, les hommes et les femmes s'accordent sur le genre qui leur est associé.

L'analyse des résultats révèle peu de variation selon l'orientation sexuelle des répondant.e.s en ce qui concerne les traits considérés comme masculins ou féminins par le BSRI (tableau 1). Les répondant.e.s hétérosexuel.le.s considèrent que le trait " enfantin.e " n'est pas un trait féminin, mais plutôt un trait neutre, tandis que chez les répondant.e.s de la diversité sexuelle, c'est le trait « loyal.e » qui est classé différemment, non comme un trait féminin, mais comme un trait neutre. Aucune différence entre les deux sous-groupes n'est observée en ce qui concerne les traits considérés comme masculins. C'est, à nouveau, parmi les traits classés comme neutres par le BSRI que des différences apparaissent. En effet, la classification des items neutres des répondant.e.s hétérosexuel.le.s ne concorde que faiblement 
avec celle des répondant.e.s de la diversité sexuelle ( $\kappa=0,34$; Landis et Koch, 1977). Pour les répondant.e.s hétérosexuel.le.s de I'UQAM, 11 des traits considérés comme neutres selon le BSRI seraient plutôt genrés, 10 étant classés comme des traits féminins et un comme un trait masculin. Les répondant.e.s de la diversité sexuelle de I'UQAM considèrent quant à eux que 13 des traits neutres selon le BSRI seraient genrés, 9 étant vus comme féminins et quatre comme masculins. Dans leur rejet de la neutralité de certains traits, les répondant.e.s hétérosexuel.le.s et de la diversité sexuelle s'entendent sur le fait que sept traits seraient plutôt féminins et un trait serait plutôt masculin. Toutefois, ils ne s'entendent pas sur la classification de huit traits neutres selon le BSRI : les répondant.e.s hétérosexuel.le.s considèrent que trois d'entre eux sont féminins alors que les répondant.e.s de la diversité sexuelle les considèrent comme des traits neutres. Inversement, les répondant.e.s de la diversité sexuelle considèrent que, parmi les traits neutres selon le BSRI, trois sont plutôt masculins et deux sont plutôt féminins, tandis que les répondant.e.s hétérosexuel.le.s considèrent ces cinq traits comme étant neutres.

\section{Discussion}

Notre étude visait à évaluer l'adéquation du BSRI avec les représentations sociales de jeunes adultes québécois. Un premier constat s'impose : la très grande majorité des traits associés par le BSRI à la féminité et à la masculinité le demeurent, ce qui indique une relative stabilité dans la codification socialement genrée des traits du BSRI. Cette stabilité n'est toutefois pas observée parmi les traits considérés comme neutres par le BSRI : 12 de ces traits sont considérés comme genrés, c'est-à-dire associés à l'un ou l'autre genre, selon les répondant.e.s de notre étude. Notre étude a donc permis, d'une part, de constater que le genre continue d'être un élément structurant des représentations normatives concernant les rôles, les comportements, les expressions et les identités, mais d'autre part, que la classification des traits neutres du BSRI ne correspond pas aux représentations sociales de genre des jeunes adultes qui fréquentent une université montréalaise. Ces derniers auraient une perception davantage genrée des traits neutres du BSRI. Par ailleurs, nous avons observé des différences dans les représentations selon le genre et l'orientation sexuelle des répondant.e.s.

Les différences mises en lumière par notre étude, entre la classification des traits neutres du BSRI et la classification de ces traits par l'ensemble des répondant.e.s de notre échantillon, pourraient s'expliquer par des changements de contexte (époque et lieu) et donc, être liées à des changements dans les représentations sociales de la féminité et de la masculinité. Ces différences touchent surtout la représentation de la féminité et peu celle de la masculinité. Les différences observées sont aussi plus nombreuses chez les répondantes que chez les répondants, les hommes ayant des représentations moins genrées que les femmes. Cette différence liée au genre du répondant pourrait être attribuable à une plus grande sensibilité des femmes aux stéréotypes dont elles peuvent être victimes. Les résultats de notre étude concordent avec l'étude de Haines et al. (2016) qui a analysé l'évolution au cours des trois dernières décennies des représentations sociales des rôles de genre chez des étudiant.e.s universitaires américains. Ces auteurs ont observé un accroissement des stéréotypes dans les rôles de genre féminins (davantage de traits sont considérés comme féminins), mais pas masculins. Ils ont aussi observé que les changements étaient plus nombreux chez les femmes que chez les hommes. Étant donné la formulation de la question utilisée par Bem, qui fait référence à ce qui est valorisé socialement et non personnellement, il est toutefois impossible de faire la distinction entre la perception des stéréotypes véhiculés dans la société et l'intériorisation de ceux-ci par les participant.e.s. 
La validité du BSRI repose notamment sur la concordance entre la classification des traits de l'instrument et les représentations sociales de genre qui prévalent dans le contexte où l'instrument est utilisé. La classification plus genrée des traits obtenue dans notre étude, comparativement à la classification des traits de l'instrument, porte à penser que l'utilisation du BSRI auprès d'une population de jeunes universitaires montréalais pourrait donner lieu à des mesures biaisées, en ce sens que le BSRI, dans sa formulation initiale, ne mesurerait pas adéquatement l'adhésion aux stéréotypes de genre tels qu'ils sont perçus actuellement par cette population. Chez les femmes, le BSRI tendrait ainsi à sous-estimer l'adhésion à des traits considérés comme féminins et à surestimer l'adhésion à des traits considérés comme neutres (ou androgynes), alors que ce biais serait beaucoup moindre, voire absent chez les hommes. Chez les personnes hétérosexuelles, le biais est similaire à celui observé chez les femmes, alors que chez les répondant.e.s de la diversité sexuelle, où une sous-estimation de la féminité est aussi présente, s'ajoute une légère sous-estimation de la masculinité. Ceci suggère que les répondant.e.s de la diversité sexuelle pourraient davantage associer certains traits aux hommes.

Le BSRI est utilisé, entre autres, dans des études sur l'effet de l'adhésion aux stéréotypes de genre, notamment des études sur la santé et le bien-être des personnes issues de la diversité sexuelle. Dans ces études, l'adhésion aux stéréotypes de genre est parfois mesurée par l'adhésion aux traits du même genre (par exemple, pour des hommes, on utilise le score de masculinité qui mesure l'adhésion aux traits considérés masculins) et parfois mesurée par l'importance des traits du genre « opposé » (par exemple, le score de féminité pour des hommes). Ainsi, des scores élevés de masculinité indiquent une plus grande adhésion subjective aux stéréotypes de genre chez les hommes et une plus grande nonadhésion aux stéréotypes de genre chez les femmes, et inversement pour les scores de féminité. Notre étude montre non seulement qu'une mesure unique pour chaque genre négligerait des différences dans la représentation des rôles de genre, mais aussi que le choix de la mesure (adhésion ou non-adhésion) pourrait induire des biais différents puisque la féminité n'est pas perçue de la même façon chez les femmes que chez les hommes.

Notre étude n'a pas pu reproduire intégralement l'étude de Bem (1974). Rappelons que le développement du BSRI a reposé sur les réponses au sujet de 400 traits de personnalité et non seulement au sujet des 60 analysés dans notre étude. II ne nous a pas été possible d'évaluer les 400 traits utilisés par Bem, car ceux-ci ne sont pas décrits dans l'article (Bem, 1974). Toutefois, notre objectif n'était pas de reproduire intégralement la démarche de Bem, mais plutôt d'analyser la concordance de la classification des items du BSRI avec les représentations sociales de jeunes adultes québécois afin d'évaluer la validité du BSRI auprès de cette population. Notre étude est, à notre connaissance, la seule à s'être penchée sur la validité du BSRI pour une utilisation auprès des jeunes adultes au Québec. La population cible de notre étude constitue une limite : l'échantillon n'étant composé que d'étudiant.e.s fréquentant une université montréalaise, il est impossible d'inférer avec assurance que les résultats valent pour l'ensemble des jeunes adultes du Québec.

Notre étude était fondée sur un échantillon de volontaires. Ce mode de collecte d'information a induit un biais dans notre échantillon, qui n'est pas représentatif de la distribution des genres au sein de la population cible. En effet, la proportion de répondantes se décrivant comme femmes est supérieure à celle qu'on retrouve dans la population d'étudiant.e.s universitaires. Bien qu'une pondération visant à corriger la surreprésentation des femmes dans l'échantillon ait été utilisée, cette surreprésentation est susceptible d'avoir affecté certains résultats ; les résultats concernant l'ensemble des répondant.e.s de I'UQAM de même que les résultats selon l'orientation sexuelle sont davantage influencés par les 
représentations sociales de genre des femmes que par celles des hommes. Soulignons toutefois que le genre des répondant.e.s n'entre pas en ligne de compte dans les différences observées entre les répondant.e.s hétérosexuel.le.s et les répondant.e.s de la diversité sexuelle puisque la distribution hommes-femmes est très similaire entre les deux groupes - celle-ci est de 23,3 \% d'hommes et 76,7 \% de femmes chez les hétérosexuel.le.s comparativement à $22,8 \%$ d'hommes et $77,2 \%$ de femmes chez les non-hétérosexuel.le.s, une différence qui n'est pas statistiquement significative $\left(\chi^{2}=0,0097\right.$, $p=0,922)$. Ainsi, l'influence de la surreprésentation des femmes sur les résultats selon l'orientation sexuelle serait sensiblement la même dans les deux groupes de répondant.e.s et ne pourrait pas expliquer de différence entre ces groupes.

À la lumière de ces résultats, il semble approprié de se pencher sur l'évaluation des représentations sociales de genre, en particulier sur la codification genrée des traits associés à l'un ou à l'autre genre ou à aucun, en tenant compte du genre des individus et de leur orientation sexuelle. Ce type de connaissances permettrait d'améliorer les instruments de mesure des représentations des stéréotypes de genre et de l'adhésion subjective à ces stéréotypes. En développant le BSRI, Bem avait le souci de ne pas décrire la masculinité et la féminité comme des pôles d'une même dimension et s'est intéressée aussi à décrire l'androgynie (traits neutres). Nos résultats montrent qu'un grand nombre de traits considérés comme neutres par le BSRI ne sont pas considérés comme tels par les participant.e.s à notre étude. Or, il est important que les instruments de mesure soient sensibles au caractère évolutif de l'attribution sociale des traits aux genres masculin ou féminin de même qu'aux différents registres d'expression du genre, notamment parce que les attributs de la masculinité et de la féminité ne sont pas perçus de la même façon selon le genre ou l'orientation sexuelle.

PHILIBERT, Mathieu, Ph. D. santé publique a, b

GAGNÉ, Andréanne, B.A. (c) sexologie a

BLANCHETTE, Émilie, B.A. (c) sexologie a

CHAMBERLAND, Line, Ph. D. sociologie a, b

a Département de sexologie, Université du Québec à Montréal b Chaire de recherche sur l'homophobie, Université du Québec à Montréal 


\section{BIBLIOGRAPHIE}

Bem, S. L. (1974). The measurement of psychological androgyny. Journal of Consulting and Clinical Psychology, 42(2), 155-162.

Conseil de recherches en sciences humaines du Canada, Conseil de recherches en sciences naturelles et en génie du Canada et Instituts de recherche en santé du Canada. (2014). Énoncé de politique des trois Conseils. Éthique de la recherche avec des êtres humains. Ottawa : Gouvernement du Canada.

Fitzpatrick, K. K., Euton, S. J., Jones, J. N., et Schmidt, N. B. (2005). Gender role, sexual orientation and suicide risk. Journal of Affective Disorders, 87(1), 35-42.

Friedman, M. S., Koeske, G. F., Silvestre, A. J., Korr, W. S., et Sites, E. W. (2006). The impact of gender-role nonconforming behavior, bullying, and social support on suicidality among gay male youth. Journal of Adolescent Health, 38(5), 621-623.

Galambos, N. L., Berenbaum, S. A., et McHale, S. M. (2009). Gender development in adolescence. Dans R. Lerner et L. Steinberg (dir.), Handbook of Adolescent Psychology I. Hoboken, NJ : John Wiley \& Sons.

Gibson, P. A., Baker, E. H., et Milner, A. N. (2016). The role of sex, gender, and education on depressive symptoms among young adults in the United States. Journal of Affective Disorders, 189, 306-313.

Haines, E. L., Deaux, K., et Lofaro, N. (2016). The times they are a-changing... or are they not? A comparison of gender stereotypes, 1983-2014. Psychology of Women Quarterly, 40(3), 353-363.

Landis J. R., et Koch, G. G. (1977). The measurement of observer agreement for categorical data. Biometrics, 33, 159-174.

Plante, I., Théorêt, M., et Favreau, O. E. (2010). Les stéréotypes de genre en mathématiques et en langues : recension critique en regard de la réussite scolaire. Revue des sciences de l'éducation, 36(2), 389-419.

Spence, J. T. (1993). Gender-related traits and gender ideology: Evidence for a multifactorial theory. Journal of Personality and Social Psychology, 64, 624-635.

Spence, J. T., et Hall, S. K. (1996). Children's gender-related self-perceptions, activity preferences, and occupational stereotypes : A test of three models of gender constructs. Sex Roles, 35, 659691.

Tsirigotis, K., Gruszczynski, W., et Tsirigotis-Maniecka, M. (2013). Gender differentiation of indirect self-destructiveness. International Journal of Occupational Medicine and Environmental Health, 26(1), 39-48. 
Tableau 1. Classification originale des items du BSRI, traduction des items et classification des items selon l'enquête menée à I'UQAM

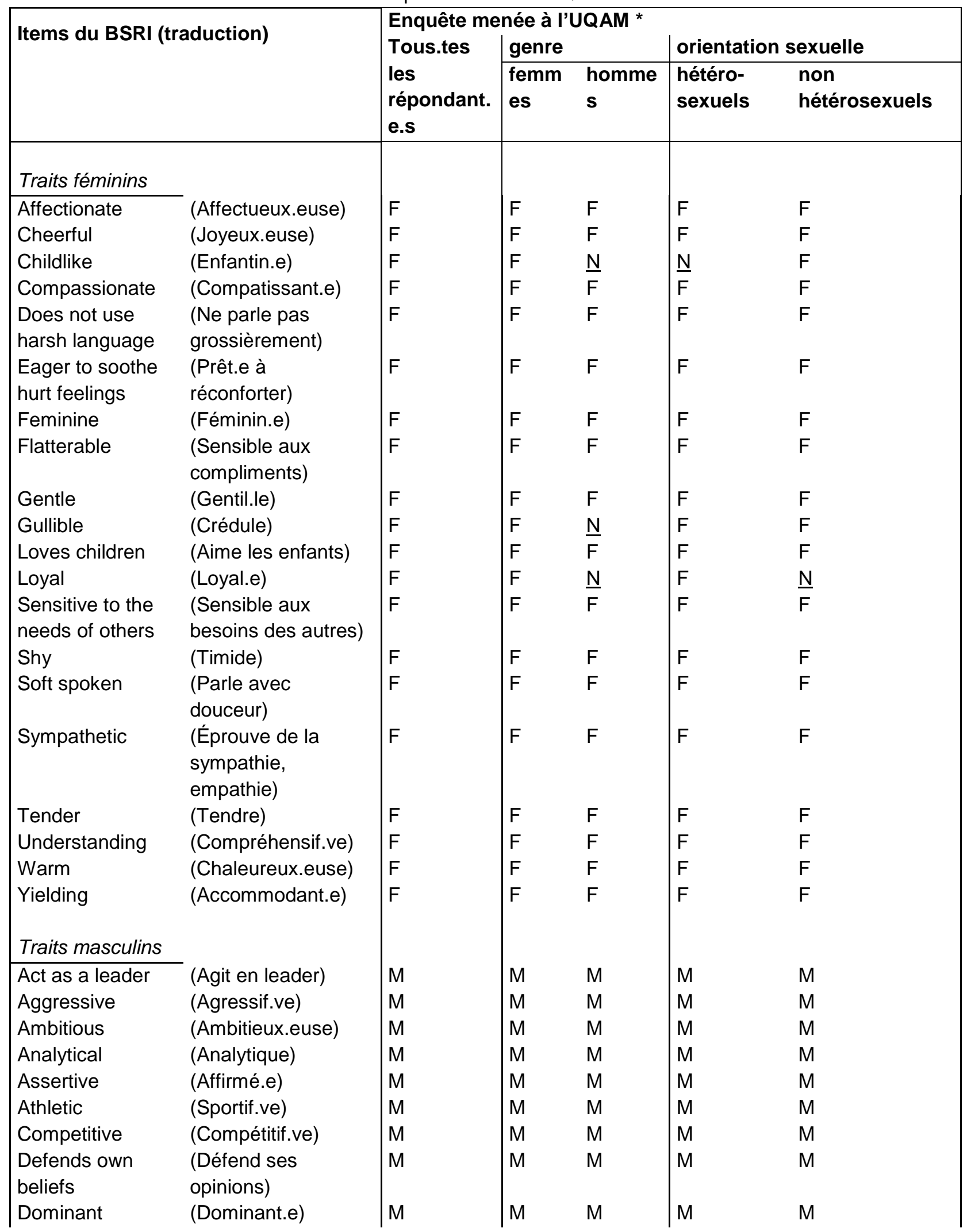




\begin{tabular}{|c|c|c|c|c|c|c|}
\hline Forceful & (Énergique) & $M$ & $M$ & $\underline{N}$ & $M$ & $M$ \\
\hline $\begin{array}{l}\text { Has leadership } \\
\text { abilities }\end{array}$ & $\begin{array}{l}\text { (A des habiletés en } \\
\text { «leadership ») }\end{array}$ & $M$ & $M$ & $\overline{\mathrm{M}}$ & $M$ & M \\
\hline Independent & (Indépendant.e) & $M$ & $M$ & $M$ & $M$ & $M$ \\
\hline Individualistic & (Individualiste) & $M$ & $M$ & $\underline{N}$ & $M$ & $M$ \\
\hline $\begin{array}{l}\text { Makes decisions } \\
\text { easily }\end{array}$ & $\begin{array}{l}\text { (Prend facilement } \\
\text { des décisions) }\end{array}$ & $M$ & $M$ & $\bar{M}$ & $M$ & $M$ \\
\hline Masculine & (Masculin.e) & $M$ & $M$ & $M$ & $M$ & $M$ \\
\hline Self-reliant & (Autonome) & $M$ & $M$ & $\underline{N}$ & $M$ & $M$ \\
\hline Self-sufficient & $\begin{array}{l}\text { (Pourvoit à ses } \\
\text { propres besoins) }\end{array}$ & $M$ & $M$ & M & $M$ & $M$ \\
\hline \begin{tabular}{|l} 
Strong \\
personality
\end{tabular} & $\begin{array}{l}\text { (A une personnalité } \\
\text { forte) }\end{array}$ & $M$ & $M$ & $M$ & $M$ & $M$ \\
\hline $\begin{array}{l}\text { Willing to take a } \\
\text { stand }\end{array}$ & $\begin{array}{l}\text { (Prêt.e à prendre } \\
\text { position) }\end{array}$ & $M$ & $M$ & $M$ & $M$ & $M$ \\
\hline $\begin{array}{l}\text { Willing to take } \\
\text { risks }\end{array}$ & $\begin{array}{l}\text { (Prêt.e à prendre } \\
\text { des risques) }\end{array}$ & $M$ & $M$ & $M$ & $M$ & $M$ \\
\hline \multicolumn{7}{|l|}{ Traits neutres } \\
\hline Adaptable & $\begin{array}{l}\text { (Capacité à } \\
\text { s'adapter) }\end{array}$ & $N$ & $N$ & $\mathrm{~N}$ & $N$ & $\mathrm{~N}$ \\
\hline Conceited & (Vaniteux.euse) & $N$ & $\underline{M}$ & $\mathrm{~N}$ & $N$ & $\underline{M}$ \\
\hline Conscientious & $\begin{array}{l}\text { (Consciencieux.eus } \\
\text { e) }\end{array}$ & $\underline{F}$ & $\underline{F}$ & $\underline{F}$ & $\underline{F}$ & $\underline{F}$ \\
\hline Conventional & (Conventionnel.le) & $N$ & $\underline{F}$ & $\mathrm{~N}$ & $N$ & $\mathrm{~N}$ \\
\hline Friendly & (Amical.e) & $\underline{F}$ & $\overline{\mathrm{F}}$ & $\mathrm{N}$ & $\underline{F}$ & $\mathrm{~N}$ \\
\hline Happy & (Heureux.euse) & $\underline{\bar{F}}$ & $\underline{\bar{F}}$ & $\mathrm{~N}$ & $\bar{N}$ & $\underline{F}$ \\
\hline Helpful & (Aidant.e) & $\overline{\bar{F}}$ & $\bar{F}$ & $\underline{F}$ & $\underline{F}$ & $\underline{F}$ \\
\hline Inefficient & (Inefficace) & $\bar{N}$ & $\bar{N}$ & $\bar{N}$ & $\bar{N}$ & $\overline{\mathrm{F}}$ \\
\hline Jealous & (Jaloux.ouse) & $N$ & $N$ & $\mathrm{~N}$ & $N$ & $\bar{N}$ \\
\hline Likable & (Aimable) & $\underline{F}$ & $\underline{F}$ & $\underline{F}$ & $\underline{F}$ & $\underline{F}$ \\
\hline Moody & $\begin{array}{l}\text { (D'humeur } \\
\text { changeante) }\end{array}$ & $\underline{\bar{F}}$ & $\underline{F}$ & $\underline{\bar{F}}$ & $\underline{F}$ & $\bar{N}$ \\
\hline Reliable & (Fiable) & $\underline{F}$ & $\underline{F}$ & $\mathrm{~N}$ & $\underline{F}$ & $\mathrm{~N}$ \\
\hline Secretive & (Cachotier.ère) & $\bar{N}$ & $\bar{N}$ & $\mathrm{~N}$ & $\bar{N}$ & $\mathrm{~N}$ \\
\hline Sincere & (Sincère) & $\underline{F}$ & $\underline{F}$ & $\underline{F}$ & $\underline{F}$ & $\underline{F}$ \\
\hline Solemn & (Solennel.le) & $\bar{N}$ & $\bar{N}$ & $\bar{N}$ & $\bar{N}$ & $\underline{M}$ \\
\hline Tactful & (A du tact) & $\underline{F}$ & $\underline{F}$ & $\underline{F}$ & $\underline{F}$ & $\underline{F}$ \\
\hline Theatrical & (Théâtral.e) & $\underline{F}$ & $\underline{F}$ & $\underline{F}$ & $\underline{F}$ & $\underline{F}$ \\
\hline Truthful & (Honnête) & $\overline{\mathrm{F}}$ & $\underline{F}$ & $\bar{N}$ & $\underline{F}$ & $\underline{F}$ \\
\hline Unpredictable & (Imprévisible) & $\bar{N}$ & $\bar{N}$ & $\mathrm{~N}$ & $\bar{N}$ & $\underline{\bar{M}}$ \\
\hline Unsystematic & (Désordonné.e) & $\underline{M}$ & $\underline{M}$ & $\underline{M}$ & $\underline{M}$ & $\underline{M}$ \\
\hline
\end{tabular}

${ }^{*} \mathrm{~F}$ : féminin, $\mathrm{M}$ : masculin, $\mathrm{N}$ : neutre

Les différences par rapport à la classification originale sont indiquées par une lettre soulignée. 